\title{
Alimentação e distribuição de piaus (Pisces, Anostomidae) na Planície do Bananal, Mato Grosso, Brasil
}

\author{
Cesar Enrique de Melo \& Cristhiana Paula Röpke
}

Departamento de Ciências Biológicas, Universidade do Estado de Mato Grosso. Campus de Nova Xavantina, Rodovia BR 148, Km 158, 78690-000 Nova Xavantina, Mato Grosso, Brasil.E-mail: meloce@yahoo.com; krikaropke@yahoo.com.br

\begin{abstract}
Feeding and distribution of piaus (Pisces, Anostomidae) in the Planície do Bananal, Mato Grosso, Brazil. The Anostomidae fishes are restricted to South America, and they are economically important to fisheries in many regions. Studies conducted in Central Amazon, where they are widely distributed, demonstrated that these fishes have high feeding plasticity. In this study we analyzed nine species of Anostomidae and we discussed ecological interactions in relation to their feeding and distribution in the Planicie do Bananal, a Cerrado region which is subject to periodic floods and it is drained by Araguaia/Tocantins basin. Niche breadth was low among the six species analyzed $(0,125$ and 0,355). Schizodon vittatum (Valencienes, 1894) has the widest niche breadth and it is also the most widely distributed species. Qualitative analyses comparing species feeding among habitats demonstrate great variation in the proportion of ingested items from one environment to another, while quantitative analyses show relatively higher similarities. Laemolyta petiti Géry, 1964 and Laemolyta taeniata (Kner 1859) are the most specialized species in relation to ingested items, as well as the proportion of each item founded in the stomach contents.

KEY WORDS. Fish ecology, Araguaia river, Cerrado, niche.
\end{abstract}

RESUMO. Os peixes da família Anostomidae, conhecidos popularmente como piaus, são restritos à América do Sul e representam importante fonte de renda para pescadores de muitas regiões. Estudos na Amazônia Central demonstram que estes peixes apresentam alta plasticidade alimentar e estão amplamente distribuídos pela região. Neste estudo, são analisadas nove espécies de Anostomidae e discutidas interações ecológicas relativas a sua alimentação e distribuição na Planície do Bananal, uma região de cerrado sujeita a inundações periódicas, drenada pela Bacia Araguaia / Tocantins. A amplitude de nicho alimentar foi baixa entre as seis espécies analisadas $(0,125$ e 0,355). Schizodon vittatum (Valencienes, 1894), a espécie com maior amplitude de nicho também é a mais amplamente distribuída. Quando se compara a alimentação das espécies entre hábitats, por análise qualitativa, ocorre grande variação na proporção dos itens ingeridos de um ambiente para outro. No entanto, quando a análise é quantitativa as similaridades são relativamente mais elevadas. Entre as espécies analisadas, Laemolyta petiti Géry, 1964 e Laemolyta taeniata $(K n e r, 1859)$ são as mais especializadas, tanto em relação aos tipos de itens ingeridos quanto à proporção de cada item presente nos estômagos.

PALAVRAS CHAVE. Ecologia de peixes, Rio Araguaia, Cerrado, nicho.

A família Anostomidae é um grupo de peixes da ordem Characiformes, composto por cerca de 110 espécies, restritas à América do Sul e com representantes em todas as bacias hidrográficas do Brasil (SANTos 1982, Nelson 1994). Conhecidos popularmente como piaus, este grupo de peixes tem elevada importância comercial e espécies de pequeno porte têm grande aceitação na aquariofilia, algumas espécies alcançam cerca de $400 \mathrm{~mm}$ de comprimento e mais de um quilo em peso (SANTOS $\&$ JEGU 1996).

Segundo Géry (1977) cerca da metade do número de espécies desta família ocorrem na bacia Amazônica. Os Anosto- mídeos da bacia Amazônica são bem conhecidos do ponto de vista taxonômico e ecológico, entretanto, ainda existem grandes lacunas de conhecimento básico, pois extensas áreas desta bacia não foram sequer amostradas. SANTOS (1981) encontrou 15 espécies deste grupo de peixes, em estudos realizados no lago Janauacá, no Amazonas. SANTOS \& Jegu (1996) na realização de um inventário taxonômico na bacia do rio Uatumã encontraram 22 espécies, sendo 12 destas pertencentes ao gênero Leporinus Agassiz, 1829. Este gênero é formado por cerca de 60 espécies (SANTOS \& JEGU 1996), englobando aproximadamente 50\% das espécies de Anostomidae. 
Na bacia Araguaia/Tocantins foram realizados poucos estudos incluindo os Anostomidae e mesmo aqueles realizados até o momento, não tiveram como objetivo central este grupo de peixes (Santos et al. 1984, Merona 1986/87, Braga 1990). A falta de conhecimento sobre os Anostomidae desta região, associado ao processo de desmatamento do cerrado, pode estar colocando em risco um dos grupos de peixes de maior importância na bacia do rio Araguaia.

As relações ecológicas básicas de uma espécie, que devem ser consideradas para seu conhecimento, podem ser resumidas em três dimensões do nicho: o nicho trófico, nicho espacial e nicho temporal (Pianka 1982). Considerando estes fatores, este trabalho tem como objetivo discutir a utilização de recursos alimentares e a distribuição de espécies de Anostomidae na Planície do Bananal, uma extensa área de cerrado sujeita a inundações periódicas, entre os rios Araguaia e Mortes, no Estado de Mato Grosso.

\section{MATERIAL E MÉTODOS}

\section{Área de estudo}

Os exemplares estudados são oriundos de ambientes lóticos e lênticos da Planície do Bananal, uma extensa planície de cerrado entre o Rio das Mortes e rio Araguaia com aproximadamente $70.000 \mathrm{~km}^{2}$, sujeita a inundação periódica. Na região predomina a vegetação de cerrado tipo savana aberta com ocorrência de campos em várias áreas (Brasil 1981). A fragilidade da vegetação facilita o desmatamento e intensifica o processo de degradação ambiental, com predominância dos grandes latifúndios para criação extensiva de gado bovino (Novaes Pinto 1993). Em muitas regiões da Planície as matas de galeria foram retiradas e substituídas por pastagem (Diegues 2002). No solo arenoso as erosões são freqüentes e alguns lagos de menor porte estão comprometidos pelo assoreamento.

Foram amostrados seis ambientes lóticos e sete ambientes lênticos, sendo eles: Córrego Capitão Décio, Córrego da Botina, Rio Corrente, Rio das Mortes, Rio Pindaíba, Rio Cristalino, Lago Diadomã, Lago dos Patos, Lago do Rosico, Lago das Piranhas, Lago Melancia, Lago Redondo e Lago Baía da Saudade (Fig. 1).

\section{Metodologia}

Os exemplares analisados foram obtidos na coleção ictiológica do Laboratório de Ictiologia e Limnologia, Departamento de Ciências Biológicas, Universidade do Estado de Mato Grosso, Campus de Nova Xavantina, coletados nos períodos de seca, cheia e vazante entre junho de 1998 a junho de 2001, com auxílio de tarrafas com malhas de 2,5 e 4,0 cm entre nós opostos; redes de arrasto com malhas de 2,0 cm e 40,0 m de comprimento por $2,5 \mathrm{~m}$ de altura; redes de espera com malhas de $3,4,5,6,7,8$, 10 e $12 \mathrm{~cm}$ entre nós opostos, com 10,0 $\mathrm{m}$ de comprimento e $1,5 \mathrm{~m}$ de altura.

Em cada um dos ambientes lênticos amostrados as redes foram expostas durante 24 horas consecutivas e revisadas a cada quatro horas para retirada dos peixes capturados. Nos

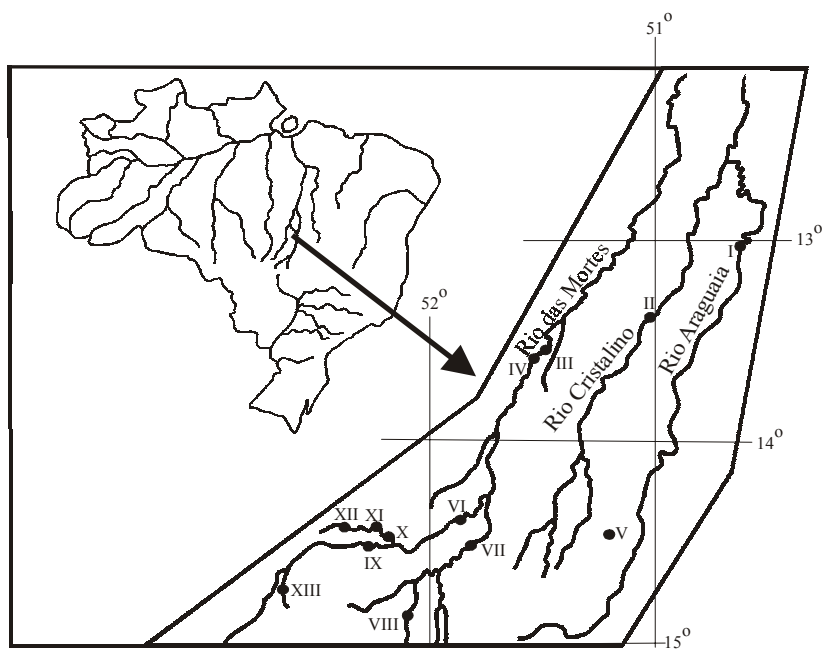

Figura 1. Mapa das localidades de amostragens na Planície do Bananal. (I) Lago das Piranhas, (II) Rio Cristalino, (III) Lago Melancia, (IV) Lago Redondo, (V) Lago Baía da Saudade, (VI) Lago Diadomã, (VII) Rio Pindaíba, (VIII) Rio Corrente, (IX) Rio das Mortes, (X) Lago do Rosico, (XI) Lago dos Patos, (XII) Córrego Capitão Décio, (XIII) Córrego da Botina.

ambientes lóticos, Rio Corrente, Rio das Mortes Rio Pindaíba e Rio Cristalino foi utilizada a mesma metodologia empregada para os lagos além do uso das redes de arrasto e nos Córregos Capitão Décio e Botina foi empregado somente o uso de tarrafas no período diurno. Todos os exemplares coletados eram fixados em campo com solução de formol a 10\% e após $72 \mathrm{~h}$, lavados e conservados em solução de etanol 70\%. Após este procedimento os exemplares foram tombados na coleção ictiológica. A identificação das espécies foi feita segundo SANTos et al. (1984), SANTOS \& JeGU (1996), BRITSKI et al. (1999) e envio de material para especialistas.

Para a determinação dos recursos alimentares utilizados pelos Anostomidae foram analisados 130 exemplares de seis espécies, sendo 50 exemplares de Schizodon vittatum (Valencienes, 1894), 26 de Laemolyta petiti Géry, 1964, 20 de Laemolyta taeniata (Kner, 1859), 20 de Leporinus affinis Günther, 1864, 12 de Leporinus friderici (Bloch, 1794) e dois de Leporinus trifasciatus Steindachner, 1876.

Para determinação da dieta alimentar dos peixes, o conteúdo estomacal foi retirado e analisado qualitativamente em placa de Petri sob estereomicroscópio ou em microscópio óptico. A medição do volume dos itens ingeridos foi adaptada da forma proposta por WootTon (1990). Para a determinação do volume do material ingerido, foram medidas a área e altura dos itens de cada categoria alimentar. A área foi medida com auxílio de papel milimetrado colocado sob a placa de Petri, onde eram realizadas as análises. Para determinação da altura, no caso de itens muito pequenos, estes eram agrupados com altura padronizada em $1 \mathrm{~mm}$. No caso de itens maio- 
res, estes eram agrupados em alturas que variavam de acordo com o tamanho do item.

No caso de itens microscópicos mediu-se o volume total do conteúdo, pelo método citado, e estimou-se, através do campo visual do microscópio óptico, a porcentagem do item em relação ao volume total. Os itens encontrados foram classificados em: a) vegetais terrestres (VT): fragmentos de madeira, raízes, folhas e flores; b) frutos (FR): frutos e sementes de vegetais superiores, ou partes destes; c) macrófitas aquáticas (MA); d) algas filamentosas (AF); e) Diatomacea (DI); f) restos orgânicos (RO): conteúdo estomacal que devido ao alto grau de digestão não foi possível a identificação; g) peixes (PX): fragmentos de músculo ou pedaços de nadadeira de peixes; $h$ ) insetos aquáticos (IA): ovos, larvas, ninfas e adultos de insetos que, quando predados, viviam restritamente em ambiente aquático; i) outros (OT): itens que, embora identificados, apresentaram baixa freqüência e volume nos estômagos, estes incluíram: algas microscópicas, insetos terrestres, protozoários e zooplâncton.

A amplitude de nicho foi determinada pelo índice de Levins padronizado $\left(B_{A}\right)$ (KreBs 1989): $B_{A}=B-1 / n-1$, onde: $\left(B_{A}\right)$ índice padronizado de Levins, (B) medida de amplitude de nicho, (n) número de recursos utilizados, (Pj) fração do item da categoria " $\mathrm{j}$ " na dieta. A similaridade alimentar entre habitas, por espécie, foi calculado pelo índice de similaridade modificado de Morisita-Horn, com as proporções dos recursos utilizados de acordo com a formula: $\mathrm{B}=1 / \Sigma \mathrm{P}^{2}$, onde $(\mathrm{CH})$ índice de similaridade modificado de Morisita-Horn; (Xij, Xik) proporção do recurso "i" no ambiente " $\mathrm{j}$ " e ambiente " $\mathrm{k}$ "; $(\mathrm{Nj}$ $=\Sigma \mathrm{Xij})$ total dos recursos no ambiente "j"; $(\mathrm{Nk}=\Sigma \mathrm{Xik})$ total dos recursos do ambiente " $k$ ", e coeficiente de similaridade de Sorensen, para ocorrência dos recursos:

$\mathrm{C}_{\mathrm{H}}=2 \sum \mathrm{X}_{\mathrm{ij}} \mathrm{X}_{\mathrm{ik}} /\left[\left(\sum \mathrm{X}_{\mathrm{ij}}^{2} / \mathrm{N}_{\mathrm{j}}^{2}\right)+\left(\sum \mathrm{X}_{\mathrm{ij}}^{2} / \mathrm{N}_{\mathrm{k}}^{2}\right) \mathrm{N}_{\mathrm{j}} \mathrm{N}_{\mathrm{k}}\right]$, onde: $\left(\mathrm{S}_{\mathrm{S}}\right)$ coeficiente de similaridade de Sorensen, (a) numero de recursos que ocorrem no ambiente " $\mathrm{A}$ " e no ambiente " $\mathrm{B}$ ", (b) número de recursos que ocorrem no ambiente "B" mas não ocorrem em " $\mathrm{A}$ ", (c) número de recursos que ocorrem no ambiente " $\mathrm{A}$ " mas não ocorrem em "B" (KREBs 1989). Com base nas proporções dos itens alimentares ingeridos foi determinada a afinidade alimentar entre as espécies, por meio de análise de cluster, pelo método UPGMA e distâncias Euclidianas, com o auxílio do programa Statistica.

\section{RESULTADOS}

Foram analisados 345 espécimes de Anostomidae, distribuídos em nove espécies (Tab. I). O gênero predominante foi Leporinus com cinco espécies, seguido por Laemolyta Cope, 1872 com duas, Schizodon Agassizi, 1829 e Anostomoides Pellegrin, 1909 com uma espécie cada. A espécie mais abundante foi Schizodon vittatum seguida por Laemolyta petiti, Leporinus affinis, Laemolyta taeniata e Leporinus friderici (Tab. I). Dentre os 13 ambientes considerados, $S$. vittatum foi a mais amplamente distribuída, ocorrendo em oito locais, seguida por $L$. friderici que ocorreu em sete.
A dieta de $S$. vittatum é composta quase exclusivamente de itens vegetais, incluindo principalmente macrófitas aquáticas, vegetais terrestres e algas filamentosas (Tab. II). Laemolyta petiti ingere principalmente restos orgânicos, vegetais terrestres e diatomáceas. Na dieta de L. taeniata ocorre preferencialmente restos orgânicos, vegetais terrestres e algas filamentosas. Porção significativa do alimento ingerido por $L$. friderici e $L$. affinis é representada por partes de musculatura de peixes. Este material foi ingerido a partir de peixes feridos nas redes, por ataques de piranhas. Além deste recurso $L$. friderici ingeriu principalmente vegetais terrestres e restos orgânicos. Além de restos de peixes, L. affinis ingeriu principalmente insetos aquáticos, ovos de insetos e restos orgânicos. Embora tenham sido analisados apenas dois indivíduos de $L$. trifasciatus estes sugerem que a espécie ingere preferencialmente frutos.

A amplitude de nicho trófico das espécies analisadas pode ser considerado baixo, entre 0,125 e 0,355 para valores possíveis entre 0 e 1 . A espécie com maior amplitude de nicho trófico é $S$. vittatum sendo também a que apresenta a mais ampla distribuição geográfica. A menor amplitude de nicho ocorre para $L$. friderici (Tab. II).

O dendrograma de agrupamento com base nos itens alimentares demonstra dois conjuntos: o primeiro, com maior afinidade, representado por $L$. taeniata e $L$. petiti e o segundo, com menor afinidade, formado por $L$. affinis e $L$. friderici (Fig. 2). Os itens alimentares com maior peso na formação do primeiro conjunto são vegetais terrestres e restos orgânicos. No segundo agrupamento o principal responsável é o item peixes. A maior distância entre as espécies deste segundo grupo se deve a ingestão relativamente importante de insetos aquáticos por L. affinis e de vegetais terrestres por $L$. friderici. Embora $S$. vittatum esteja mais relacionado ao grupo composto por espécies de Laemolyta, a ligação ocorre por distância relativamente grande, podendo neste caso ser caracterizado como distinto do primeiro.

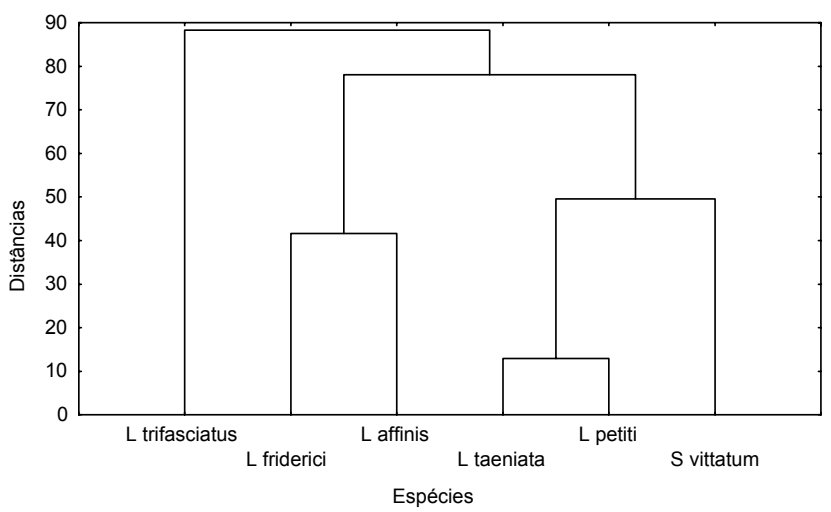

Figura 2. Dendrograma de agrupamento para seis espécies de Anostomidae, com base nos itens alimentares ingeridos.

De forma geral, as análises qualitativas, que envolvem apenas as categorias alimentares, sem considerar a proporção

Revista Brasileira de Zoologia 21 (1): 51-56, março 2004 
Tabela I. Distribuição e abundância ( $n$ ) das espécies de Anostomidae coletadas na Planície do Bananal.

\begin{tabular}{|c|c|c|c|c|c|c|c|c|c|c|c|c|c|c|}
\hline \multirow[t]{2}{*}{ Espécie } & \multicolumn{13}{|c|}{ Locais Amostrados } & \multirow[b]{2}{*}{$\mathrm{N}$} \\
\hline & 1 & II & III & IV & $\mathrm{V}$ & $\mathrm{VI}$ & VII & VIII & IX & $x$ & $\mathrm{XI}$ & XII & XIII & \\
\hline S. vittatum (Valencienes, 1894) & & & 21 & 7 & & 79 & 4 & 7 & 1 & 2 & 22 & & & 143 \\
\hline L. petiti Géry, 1964 & & & 36 & 10 & 3 & 7 & 1 & & & & 1 & & & 58 \\
\hline L. taeniata (Kner, 1859) & & & 19 & 3 & & 4 & & 18 & 2 & & & & & 46 \\
\hline L. affinis Günther, 1864 & & 2 & 32 & 17 & 1 & & & 1 & & 1 & & & & 54 \\
\hline L. friderici (Bloch, 1794) & 1 & & 9 & 9 & 2 & & & 1 & & 2 & & 3 & & 27 \\
\hline L. trifasciatus Steindachner, 1876 & & & 1 & 2 & & & & & 5 & & & & & 8 \\
\hline L. megalepis Günther, 1863 & & & & & & & & 1 & & & & & 2 & 3 \\
\hline L. agassizi Steidachner, 1876 & & & 1 & & & & & & & & & & 2 & 3 \\
\hline A. laticeps (Eigenmann,1912) & & & 1 & & & & & & & & & & & 1 \\
\hline
\end{tabular}

Tabela II. Volume relativo dos itens alimentares ingeridos, número de indivíduos analisados ( $\mathrm{n}$ ) e coeficientes de amplitude de nicho alimentar (BA), para seis espécies de Anostomidae da Planície do Bananal. (MA) macrófitas aquáticas, (VT) vegetais terrestres, (AF) algas filamentosas, (RO) restos orgânicos, (DI) diatomáceas, (IA) insetos aquáticos, (FR) frutos, (PX) peixes, (OT) outros.

\begin{tabular}{|c|c|c|c|c|c|c|c|c|c|c|c|}
\hline \multirow{2}{*}{ Espécie } & \multicolumn{9}{|c|}{ Itens Alimentares (\%) } & \multirow{2}{*}{$\mathrm{n}$} & \multirow{2}{*}{$\mathrm{B}_{\mathrm{A}}$} \\
\hline & MA & VT & $\mathrm{AF}$ & $\mathrm{RO}$ & $\mathrm{Dl}$ & IA & FR & PX & OT & & \\
\hline S. vittatum & 33,50 & 26,50 & 17,00 & 15,60 & 3,40 & 2,40 & 1,60 & - & - & 50 & 0,355 \\
\hline L. petiti & - & 38,43 & 0,33 & 48,70 & 9,89 & 0,15 & 1,30 & - & 1,20 & 26 & 0,168 \\
\hline L. taeniata & - & 37,83 & 10,15 & 46,04 & 2,30 & 0,25 & 2,93 & - & 0,50 & 20 & 0,191 \\
\hline L. affinis & - & 2,70 & 0,08 & 4,80 & 0,36 & 36,08 & 1,78 & 54,10 & 0,10 & 20 & 0,150 \\
\hline L. friderici & - & 26,70 & - & 9,70 & - & 0,20 & 1,10 & 62,30 & - & 12 & 0,125 \\
\hline L. trifasciatus & - & - & - & 30,00 & - & - & 70,00 & - & - & 2 & - \\
\hline
\end{tabular}

Tabela III. Índices de similaridades qualitativos (quadros cinzas) e quantitativos, com base nos itens alimentares ingeridos, para cinco espécies de Anostomidae coletados em diversos ambientes da Planície do Bananal, Mato Grosso. (LM) Lago Melancia, (LD) Lago Diadomã, (RC) Rio Correntes, (LP) Lago dos Patos, (LR) Lago Redondo, (LRo) Lago do Rosico, (Sv) Schizodon vittatum, (La) Leporinus affinis, (Lp) Laemolita petiti, (Lf) Leporinus friderici, (Lt) Laemolita taeniata. Espécies ausentes desta tabela ou ambientes sem indicação de valores de índices, não apresentaram número suficiente de indivíduos para realização deste tipo de análise.

\begin{tabular}{|c|c|c|c|c|c|c|c|}
\hline \multirow{2}{*}{\multicolumn{2}{|c|}{ Espécies }} & \multicolumn{6}{|c|}{ Ambientes } \\
\hline & & LM & LD & $\mathrm{RC}$ & $L P$ & $\mathrm{LR}$ & LRo \\
\hline \multirow{4}{*}{ Sv } & LM & & 0,450 & 0,460 & 0,850 & & \\
\hline & LD & 0,909 & & 0,580 & 0,270 & & \\
\hline & $\mathrm{RC}$ & 0,833 & 0,909 & & 0,260 & & \\
\hline & LP & 0,286 & 0,333 & 0,286 & & & \\
\hline \multirow{2}{*}{ La } & LM & & & & & 0,280 & \\
\hline & LR & 0,923 & & & & & \\
\hline \multirow{3}{*}{ Lp } & LM & & 1,000 & & & 0,950 & \\
\hline & LR & 0,727 & 0,960 & & & & \\
\hline & LD & 0,600 & & & & 0,857 & \\
\hline \multirow{3}{*}{ Lf } & LM & & & & & 1,000 & 0,160 \\
\hline & LR & 1,000 & & & & & 0,120 \\
\hline & LRo & 0,667 & & & & 0,667 & \\
\hline \multirow{2}{*}{$\mathrm{Lt}$} & $\mathrm{RC}$ & 0,950 & & & & & \\
\hline & LM & & & 0,923 & & & \\
\hline
\end{tabular}

Revista Brasileira de Zoologia 21 (1): 51-56, março 2004 
de cada item, apresentaram valores de similaridades um pouco superiores aos valores encontrados nas análises quantitativas, que consideram as proporções dos itens ingeridos.

No caso de $S$. vittatum, a análise de similaridade qualitativa da alimentação obtida no Lago Melancia, Rio Corrente e Lago Diadomã, indica altos níveis de similaridade (Tab. III). No entanto, o mesmo tipo de análise demonstra que o alimento ingerido no Lago dos Patos, difere acentuadamente de todas os outros ambientes. Na análise quantitativa, apenas uma relação pode ser considerada como de alta similaridade, entre Lago Melancia e Lago dos Patos. Em ambos, a alimentação se baseou em um item principal, que representou proporção significativa da dieta da espécie. Outros itens, foram consumidos em menor escala e variaram consideravelmente entre estes ambientes, determinando os baixos índices na análise qualitativa.

Entre as espécies analisadas, apenas L. petiti e L taeniata apresentaram índices de similaridades elevados para as análises qualitativas e quantitativas entre todos os ambientes. No caso de L. taeniata, este índice ainda é muito elevado mesmo quando comparados ambientes lênticos como Lago Melancia e lótico, como o Rio Corrente.

\section{DISCUSSÃO}

Segundo Goulding (1980) os peixes da família Anostomidae têm hábitos alimentares generalistas e o tipo de alimento ingerido depende da disponibilidade destes no hábitat. Esta estratégia alimentar favorece espécies amplamente distribuídas pois permite a ocupação de hábitats ecologicamente distintos (Lowe-McConNell 1999). De forma geral, esta afirmativa corrobora os dados obtidos para os Anostomidae da Planície do Bananal. Schizodon vittatum, a espécie mais abundante deste grupo, embora seja predominantemente herbívora, como descrita por SANTOS et al. (1984) e BRAGA (1990) no Rio Tocantins e FERREIRA et al. (1998) no médio Rio Amazonas, utiliza maior variedade de recursos na alimentação. A maior amplitude de nicho trófico desta espécie sugere que esta estratégia alimentar pode ser a responsável pela ampla distribuição de S. vittatum na Planície do Bananal.

Resultados obtidos por Braga (1990) demonstram que Laemolyta petiti utiliza principalmente detritos e vegetais superiores na alimentação. Na Planície do Bananal, tanto L. petiti como L. taeniata não utilizam detritos em suas dietas, no entanto, vegetais superiores são predominantes, além de perifiton.

A utilização de perifiton na alimentação de L. petiti também é relatada por SANTOS et al. (1984). A ingestão de quantidades significativas de perifiton, representadas por algas filamentosas e diatomáceas, ou mesmo detritos, como relatados em outros estudos, é reflexo de adaptações morfológicas deste grupo, cuja forma da boca e dentes estão adaptados para raspar superfícies. Lowe-McConnell (1999) descreve que no Rio Trombetas L. taeniata utiliza esponjas como um importante item na dieta. Neste caso, esponjas ocorrem em micro- habitats similares àqueles onde Laemolyta encontra algas filamentosas e diatomáceas, geralmente superfícies de troncos ou rochas submersas. Isto sugere que embora as espécies de Anostomidae possam ser generalistas, a utilização dos recursos alimentares é limitada por adaptações de cada grupo.

Vários estudos demonstram que $L$. friderici e $L$. affinis têm uma dieta com proporções elevadas de itens de origem animal, representados principalmente por insetos e fases larvais destes (Santos 1981, Santos et al. 1984, Braga 1990, Ferreira et al. 1998). Este último autor inclui L. affinis como espécie carnívora, utilizando invertebrados na sua dieta. Embora Meschiatti (1995) classifique $L$. friderici como herbívora, cita que escamas de peixes e insetos são itens importantes na sua dieta.

Embora as amplitudes de nichos tróficos de $L$. friderici e L. affinis sejam relativamente baixas, é provável que esta espécie tenha como estratégia alimentar o oportunismo, utilizando o item alimentar que esteja disponível. Segundo LoweMcConnell (1999) na bacia Amazônica os peixes de modo geral são oportunistas, com algum grau de preferência. O hábito de ingerir musculatura de peixes por estas espécies, conforme descrito também por GouLDING (1980) e BraGA (1990) em outras regiões, confirma a estratégia alimentar oportunista. Segundo Goulding et al. (1988) isto favorece as espécies em ambientes altamente sazonais. Na planície do Bananal os períodos de seca e cheia muito acentuados (Brasil 1981), torna os recursos disponíveis na seca muito distintos daqueles da cheia, obrigando as espécies de peixes a explorar hábitats muito distintos entre um período e outro, beneficiando espécies com esta estratégia alimentar.

Quando se compara a alimentação das espécies entre habitats, fica claro que, na maioria dos casos, ocorre grande variação na proporção dos itens ingeridos de um ambiente para outro, como demonstrado de forma geral pelos menores valores de similaridades obtidos pelos índices quantitativos. No entanto, quando a análise se dá pela ocorrência do item, independente da proporção que este representa no total ingerido pela espécie, as similaridades são relativamente mais elevadas. Esta relação corrobora os dados obtidos pela análise de amplitude de nicho, indicando que estas espécies utilizam poucos itens na alimentação e estes representam a base alimentar da espécie, independente do local onde ocorram. As diferenças na alimentação entre habitats é muito mais pela proporção de cada item do que pelo tipo de alimento ingerido. Embora a disponibilidade dos recursos no ambiente não tenha sido analisada, os resultados sugerem que a proporção dos recursos utilizados é reflexo da disponibilidade do alimento no ambiente e do grau de especialização da espécie (Winemiller 1996).

Segundo Winemiller (1992) a ecologia alimentar está correlacionada com aspectos morfológicos como formato dos dentes, tamanho da boca e orientação bucal. A alta similaridade alimentar entre hábitats nas análises que envolveram $L$. petiti e L. taeniata, confirma esta proposição. A boca voltada para cima e os dentes planos e mais desenvolvidos na maxila 
inferior, nas duas espécies, permite a raspagem de substratos e a ingestão dos microorganismos em micro-habitats similares, mesmo em ambientes distintos, como sistemas lênticos ou lóticos. Quando estes itens são classificados em categorias amplas, como as adotadas aqui, é provável que a coincidência entre estes seja muito elevada. Além disso, os micro-habitats explorados, mesmo em sistemas distintos, como lênticos e lóticos, podem apresentar similaridades, como troncos submersos, pontas de raízes marginais ou material originário das matas marginais. Entre as espécies analisadas, $L$. petiti e $L$. taeniata também são as mais especializadas em relação ao alimento ingerido, e as mais distintas dos outros grupos, em relação à morfologia bucal.

Embora os peixes da família Anostomidae demonstrem alta plasticidade alimentar e ampla distribuição na Planície do Bananal, a estreita relação entre seu alimento e as regiões marginais dos ambientes aquáticos pode estar colocando em risco o futuro deste grupo.

Um dos componentes mais importantes para a manutenção da qualidade do habitat é a mata de galeria (WeBsTer \& WAIDE 1982). A retirada desta vegetação elimina a entrada de restos vegetais no sistema, como galhos e caules, que diversificam a estrutura do hábitat e servem de substrato para perifíton e invertebrados aquáticos e fornece frutos, sementes e flores para a alimentação deste grupo. A atual forma de exploração do solo nesta região, sem planejamento que vise a conservação dos ecossistemas aquáticos, coloca em risco os corpos d'água e em conseqüência grupos extremamente importantes de peixes locais, inclusive aqueles com importância econômica, como os Anostomidae.

\section{REFERÊNCIAS BIBLIOGRÁFICAS}

Braga, F.M. DE S. 1990. Aspectos da Reprodução e Alimentação de Peixes comuns em um trecho do Rio Tocantins entre Imperatriz e Estreito, Estado do Maranhão e Tocantins, Brasil. Revista Brasileira de Biologia, Rio de Janeiro, 50 (3): 547-556.

Brasil, Ministério das Minas e Energia. 1981. Projeto RADAMBRASIL. Rio de Janeiro, 640p.

Britski, H.A.; Z. De S. DE Silimon; B.S. Lopes. 1999. Peixes do Pantanal: manual de identificação. Brasília, Embrapa, 184p.

Diegues, A.C. 2002. Médio Curso do Rio Araguaia e Ilha do Bananal, p. 210-216. In: A.C. Diegues (Ed.). Povos e Águas: inventário de áreas úmidas brasileiras. São Paulo, NUPAUB, USP, $2^{\text {a }}$ ed., 597p.

Ferreira, E.J.G.; J.A. S. Zuanon \& G.M. dos Santos. 1998. Peixes Comerciais do Médio Amazonas: Região de Santarém, Pará. Brasília, Ibama, 211p.

GérY, J. 1977. Characoids of the world. Neptune City, TFH Publishing, 672p.

Goulding, M. 1980. The Fishes and the forest: explorations in Amazonian natural history. California, University of
California Press, XI $+280 p$.

Goulding, M.; M.L. Carvalho \& E.G. Ferreira. 1988. Rio Negro, rich life in poor water: Amazonian diversity and foodchain ecology as seen thought fish communites. California, SPB Academic Publishing, XI+200p.

KreBS, C.J. 1989. Ecological Methodology. New York, Harper Collins Publishers, XII+654p.

Lowe-McConnell, R.H. 1999. Estudos Ecológicos de Comunidades de Peixes Tropicais. São Paulo, USP, 535p.

Merona, B. DE. 1986/87. Aspectos Ecológicos da Ictiofauna do Baixo Tocantins. Acta Amazonica, Manaus, 16/17: 109124.

Meschiatti, A.J. 1995. Alimentação da Comunidade de Peixes de uma Lagoa Marginal do Rio Mogi-Guaçú, SP. Acta Limnológica Brasiliensis, Botucatu, 7: 115-137.

Nelson, J.S. 1994. Fishes of the world. New York, John Wiley $\&$ Sons, $3^{\text {rd }}$ ed., XVII + 600p.

Novaes PINTO, M. 1993. Cerrado: caracterização, ocupação e perspectivas. Brasília, Edunb, $2^{\mathrm{a}}, 681 \mathrm{p}$.

PIANKA, E.R. 1982. Ecologia Evolutiva. Barcelona, Omega, 365p.

SANTOS, G.M. Dos. 1981. Estudos de Alimentares e Hábitos alimentares de Schizodon fasciatus Agasiz, 1829, Rhytiodus microlepis Kner, 1859 e Rhytiodus argenteofuscus Kner, 1859, do Lago Janauacá - AM. (Osteichthyes, Characoidei, Anostomidae). Acta Amazônica, Manaus, 11 (2): 267-283.

ـ 1982. Caracterização, hábitos alimentares e reprodutivos de quatro espécies de "Aracus" e considerações sobre o grupo no lago Janauacá - AM. (Osteichtyes, Characoidei, Anostomidae). Acta Amazônica, Manaus, 12 (4): 713-739.

SAntos, G.M. dos; M. Jegu, \& B. de Merona, 1984. Catálogo de Peixes Comerciais do Baixo Rio Tocantins: Projeto Tucuruí. Manaus: Eletronorte, INPA, 83p.

SAnTos, G.M. dos \& M. Jegu. 1996. Inventário Taxonômico dos Anostomideos (Pisces, Anostomidae) da Bacia do Rio Uatumã - AM, Brasil, com descrição de duas Espécies Novas. Acta Amazônica, Manaus, 26: 151-184.

WEBSTER, J.R. \& J.B. WAIDE. 1982. Effects of the forest clearcutting on leaf breakdown in a southern Appalachian stream. Freshwater Biology, Oxford, 12: 331-344.

Winemiller, K.O. 1992. Ecomorphology of Freshwater Fisher. National Geographic Resarch \& Explorations, Washington, 8 (3): 308-327.

- 1996. Factors Driving Temporal and Spatial Variation in Aquatic Floodplain Food Webs, p. 298-312. In: G.A. Polis \& K.O. Winemiller (Eds). Food Webs: integration of patterns \& dynamics. London, Chapman \& Hall, 846p.

Wooton, R.J. 1990. Ecology of Teleost Fishes. New York, Chapman and Hall, 404p.

Recebido em 27.VI.2003; aceito em 20.I.2004.

Revista Brasileira de Zoologia 21 (1): 51-56, março 2004 